\title{
SRA: A NEW ION ACCELERATOR SCHEME
}

Joon $Y$. Choe and Han S. Uhm

Naval Surface Weapons Center, White Oak, Silver Spring, Maryland 20910

\section{SUMMARY}

The space-charge resonance accelerator (SRA) consists of a relativistic electron beam propagating through a dielectric loaded drift tube. In a range of physical parameters, the phase velocity of a selfgrowing space-charge wave increases slowly from zero to a large beam velocity as it propagates into the downstream region, thereby trapping and accelerating ions by its electric field. The self-growing mechanism of the space-charge wave is a typical Cherenkov radiation.

In recent years, a number of collective ion acceleration methods with 1 inear electron beams have been proposed and investigated at several laboratories.1-5 One of the successful experiments in collective ion acceleration is the linear beam ion acceleration in a evacuated drift tube where ions are provided by an insulating material or a local gas puff. 4,5 In this paper, we present a new promising scheme to accelerate ions by utilizing relativistic electron beams. A schematic system configuration of the space-charge resonance accelerator (SRA) is presented in Fig. 1(a), where a relativistic electron beam with radius $R_{b}$ enters into a cylindrical drift tube loaded with a dielectric material in the range $\mathrm{R}_{\mathrm{w}}<\mathrm{r}<\mathrm{R}_{\mathrm{c}}$. The dielectric constant of the dielectric material is denoted by $\varepsilon$. A grounded cylindrical conducting wall is located at radius $R_{c}$, which in

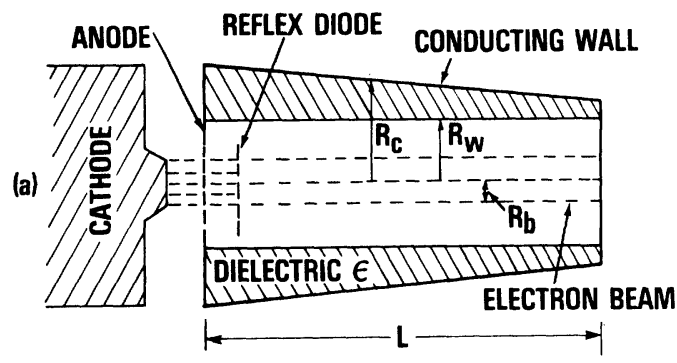

(b)

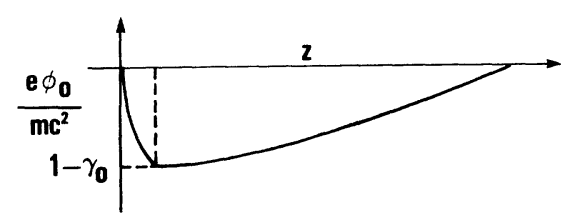

(c)

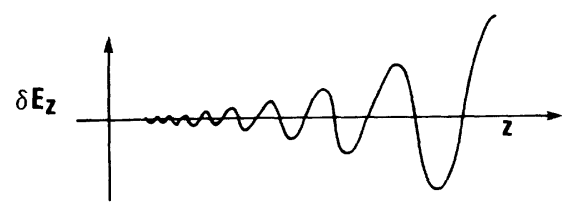

Fig. 1. Schematic drawings of the space-charge resonance accelerator: (a) System configuration, (b) Plot of the normalized potential versus $z$, (c) Growth of the space-charge wave by instability mechanism. general is a function of the axial coordinate $z$. The electron energy at the anode is $\gamma_{0} \mathrm{mc}^{2}$ where $\mathrm{m}$ is the rest mass of electrons and $c$ is the speed of light in vacuo. A reflex diode mesh is located at the position where the virtual cathode occurs. The drift tube length is denoted by L. A strong, externally applied magnetic field is needed to confine the beam electrons radially.

The formation of a virtual cathode downstream of the anode occurs where the injected electron beam current at the anode exceeds the limiting current for propagation of an electron beam, reflecting back most of the electrons at the reflex diode mesh. In this regard, only a small fraction of beam electrons can propagate further into the downstream region. The electric potential $\phi(r, z)$ in the drift tube can be self-consistently determined from the Poisson equation,

$$
\left(\frac{1}{r} \frac{\partial}{\partial r} r \frac{\partial}{\partial r}-\frac{\partial^{2}}{\partial z^{2}}\right) \phi(r, z)=-4 \pi \rho(r, z),
$$

including influence of the geometric configuration. In Eq. (1), $\rho(r, z)$ is the charge density. However, calculation of the electric potential $\phi(r, z)$ is rather a formidable task. In the case when the potential variation in the axial direction is dominant (i.e., $|\partial \phi / \partial z| \gg|\partial \phi / \partial \mathbf{r}|)$, Eq. (1) reduces to a one-

dimensional Poisson equation which has been extensive$1 y$ investigated in the previous literatures. 5,6 obviously, the distance $L$ between the anode plane and the conducting plane at end of the drift tube plays a major role in the potential determination.

On the other hand, when the potential variation in the radial direction is dominant, Eq. (1) can be approximated by

$$
\frac{1}{r} \frac{\partial}{\partial r} r \frac{\partial}{\partial r} \phi(r)=\frac{4 I}{R_{b}^{2} \beta c} U\left(R_{b}-r\right),
$$

where $I$ is the total current, $B C$ is the axial velocity of electrons at axis and $U(x)$ is the Heaviside step function. In obtaining Eq. (2), we assume that the current density is uniform over the beam cross section consistent with a thin heam. Defining $\phi_{0}=\phi(r=0)$ and making use of the identity $\gamma=\gamma_{0}+\mathrm{e} \phi_{0} / \mathrm{mc}^{2}$, where $\gamma \mathrm{mc}^{2}$ is the electron energy at axis and $-\mathrm{e}$ is the electron charge, it is straightforward to show

$$
\begin{aligned}
f(\gamma) & \equiv \frac{\left(\gamma^{2}-1\right)\left(\gamma-\gamma_{0}\right)^{2}}{\gamma^{2}} \\
& =\left(\frac{e I}{m c^{3}}\right)^{2}\left\{1+2\left(\ln \left(\frac{R_{c}}{R_{b}}\right)-\frac{\varepsilon-1}{\varepsilon} \ln \left(\frac{R_{c}}{R_{w}}\right)\right)\right\} .
\end{aligned}
$$

After careful examination of Eq. (3), we find that the function $f(\gamma)$ increases from zero to its maximum value $\left(\gamma_{0}^{2} / 3\right)^{3}$ as the $\gamma$ value decreases from $\gamma=\gamma_{0}$ to $\gamma=\gamma_{0}^{1 / 3}$. In this context, the maximum current (or limiting current) can be expressed as 


$$
I_{\max }=\frac{\left(\mathrm{mc}^{3} / \mathrm{e}\right)\left(\gamma_{0}^{2 / 3}-1\right)^{3 / 2}}{1+2\left[\ln \left(\mathrm{R}_{\mathrm{c}} / \mathrm{R}_{\mathrm{b}}\right)-(\varepsilon-1) \ln \left(\mathrm{R}_{\mathrm{c}} / \mathrm{R}_{\mathrm{w}}\right) / \varepsilon\right]} .
$$

From Eq. (3), it is found that the axial velocity $B C$ of electrons is an increasing function of the dielectric constant $\varepsilon$ and the ratio $R_{c} / R_{w}$. Moreover, Eq. (4) is identical to the result obtained by Bogdankevich and Rukhadze ${ }^{7}$ in the limit of $\varepsilon=1$ or $\mathrm{R}_{\mathrm{W}} / \mathrm{R}_{\mathrm{C}}=1$. Apparently, the electric potential profile is determined from the combination of these two extreme cases. Shown in Fig. 1(b) is a schematic plot of the normalized potential $\mathrm{e}_{0}(\mathrm{z}) / \mathrm{mc}^{2}$ versus $\mathrm{z}$. It is worthy to note from Fig. 1(b) that the axial velocity $B c$ of electrons is monotonically increasing from zero to $\left(\gamma_{0}^{2}-1\right)^{1 / 2} c / \gamma_{0}$ as the electrons move from the location of the reflex diode mesh to $z=L$. In general, the velocity profile $\beta(z) c$ is described in terms of the parameters $L, \varepsilon$, and $R_{W} / R_{C}$.

It has been shown in the previous study $^{8}$ that the slow space-charge wave couples with the transverse magnetic (TM) dielectric waveguide mode, exhibiting a strong instability. The physical mechanism of instability is the well-known Cherenkov radiation. In an unstable range of physical parameters, amplitude of the slow space-charge wave grows as it propagates into the downstream region [see Fig. 1(c)]. The TM dielectric waveguide mode is obtained from the differential equation

$$
\left(\frac{1}{r} \frac{\partial}{\partial r} r \frac{\partial}{\partial r}+p^{2}\right) \delta E_{z}(r)=0,
$$

where $\delta E$ is the axial component of the electric waveguide fieldd, and $\mathrm{p}^{2}=\omega^{2} \varepsilon / \mathrm{c}^{2}-\mathrm{k}^{2}$ for $\mathrm{R}_{\mathrm{w}}<\mathrm{r}<\mathrm{R}_{\mathrm{c}}$ and $\mathrm{p}^{2}=\omega^{2} / \mathrm{c}^{2}-\mathrm{k}^{2}$ for $0 \leq \mathrm{r}<\mathrm{R}_{\mathrm{w}}$, $\omega$ is the eigenfrequency and $\mathrm{k}$ is the axial wavenumber. In general, solutions to Eq. (5) are a linear combination of Bessel functions of the first kind $J_{\ell}(x)$ and second kind $N_{\ell}(x)$ of order l. After a straightforward algebra, we obtain the TM dielectric waveguide mode,

$$
\frac{\xi J_{0}(\xi)}{J_{1}(\xi)}=\frac{\eta}{\varepsilon} \frac{J_{0}(\eta) N_{0}(\zeta)-J_{0}(\zeta) N_{0}(\eta)}{J_{1}(\eta) N_{0}(\zeta)-J_{0}(\zeta) N_{1}(n)},
$$

where the parameters $\varepsilon, n$, and $\zeta$ are defined by $\omega^{2} / \mathrm{c}^{2}-\mathrm{k}^{2}=\xi^{2} / \mathrm{R}_{\mathrm{w}}^{2}, \omega^{2} \varepsilon / \mathrm{c}^{2}-\mathrm{k}^{2}=n^{2} / \mathrm{R}_{\mathrm{w}}^{2}$ and $\zeta={ }_{n} R_{\mathrm{c}} / \mathrm{R}_{\mathrm{w}}$.

Maximum coupling of the space-charge wave and the dielectric waveguide mode occurs near the intersecting point of the free streaming mode,

$$
\omega=\mathrm{k} \beta \mathrm{c},
$$

with the waveguide mode in Eq. (6). In order for a steady growth of the space-charge wave, it is necessary to find the conditions for the maximum coupling. Solving simultaneously Eqs. (6) and (7) for a broad range of physical parameters, we present in Fig. 2 plots of the beam velocity $\beta c$ versus $R_{w} / R_{c}$ corresponding to the maximum coupling for $\left(\omega R_{W} / c\right)^{2}=1$ and 4 , and several different values of the dielectric constant $\varepsilon$. For $R_{W} / R_{c} \ll 1$, Eq. (6) can be approximated by $\omega^{2} \varepsilon / c^{2}-k^{2}=\beta_{01}^{2} / R_{c}^{2}$ where $\beta_{01}$ is the first root of $\mathrm{J}_{0}\left(B_{01}\right)=0$. Therefore, the maximum coupling condition is given by

$$
\beta=\left[\varepsilon-\beta_{01}^{2} /\left(\omega R_{c} / c\right)^{2}\right]^{-1 / 2},
$$

which agrees excellently with the plots in Fig. 2 for $\mathrm{R}_{\mathrm{W}} / \mathrm{R}_{\mathrm{c}} \leq 0.4$. Velocity of the electron beam after the reflex diode increases as it propagates further into the downstream region. In this regard, for specified values of $\omega R_{w} / c$ and $\varepsilon$, contouring the conducting wall according to Fig. 2 gives a maximum growth of the space-charge wave all the way through the downstream region. On the other hand, we note from Fig. 2 that the maximum growth can also be attainable by changing the dielectric constant $\varepsilon$ along the $z$-direction instead of contouring of the wall.

Finally, the phase velocity $\omega / k$ for the slow space-charge wave is determined from $^{8}$

$$
\left(\frac{k \beta c}{\omega}-1\right)^{2}=\frac{4 v}{\gamma^{3}} \frac{1-\left(\frac{\omega}{k c}\right)^{2}}{\left(\frac{\omega c}{k c}\right)^{2}+\left(\frac{\omega R}{c}\right)^{2}\left(1-\frac{\omega}{k^{2} c^{2}}\right)}
$$

where $\nu=\mathrm{N}_{\mathrm{b}} \mathrm{e}^{2} / \mathrm{mc}^{2}$ is Budker's parameter of the beam, $g_{f}$ is a geometric factor of order unity, and $N_{b}$ is the number of electrons per unit axial length. Note that $\mathrm{N}_{\mathrm{b}}$ has a maximum value near the reflex diode and decreases as the beam propagates further into the downstream region. In this regard, even for a small Budker's parameter averaged over the entire beam in the drift tube, the local value of Budker's parameter near the reflex diode can be very large.

For a small beam velocity $(\beta \ll 1)$, corresponding to the beam segment right after the reflex diode in Fig. 1(a), Eq. (9) can be approximated by

$$
\omega / k c \simeq B\left[1+2\left(c / \omega R_{b}\right) \nu^{1 / 2}\right]^{-1},
$$

indicating that the phase velocity of the space-charge wave is a small fraction of the beam velocity for

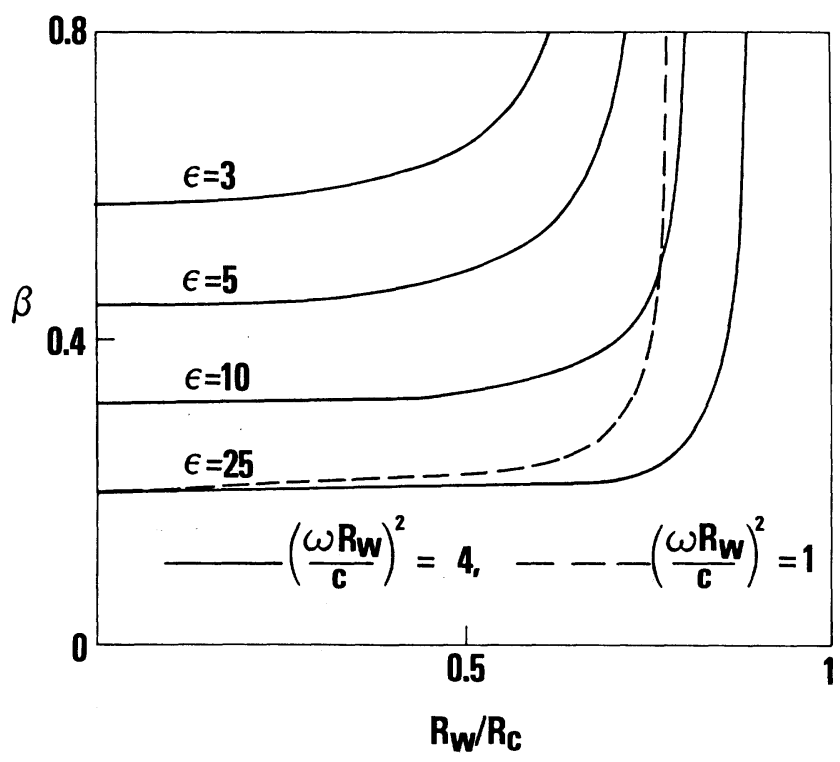

Fig. 2 Plots of the beam velocity $\beta c$ versus $R_{W} / R_{C}$ corresponding to the maximum coupling for $\left(\omega R_{W} / c\right)^{2}=1$ and 4 , and several different values of dielectric constant $\varepsilon$. 
Budker's parameter order unity. Obviously, Budker's parameter $v=I_{b} / I_{A} \beta$ is very large for small beam velocity $(\beta \ll 1)$. Here $I_{b}$ and $I_{A}=17$ Kiloamperes are the beam and Alfven currents, respectively. For example, for $\beta=0.2, \mathrm{R}_{\mathrm{b}} \omega / \mathrm{c}=0.5$ and $\nu=1$, the phase velocity of the space-charge wave is given by $\omega / \mathrm{kc}=$ 0.04 which is already sufficiently small to initially trap and accelerate ions. However, we note from Eq. (9) that the phase velocity of the space-charge wave approaches to the beam velocity as $\beta$ increases to unity, clearly indicating advantages in the collective ion acceleration. Ions, initially trapped near the reflex diode by the space-charge wave, are further accelerated by the wave electric field. In order to achieve a maximum ion acceleration, the ion velocity is synchronized with the phase velocity of the space-charge wave. Meanwhile, the amplitude of the space-charge wave is steadily growing by the instability mechanism, providing a necessary energy for ion acceleration. The unstable mechanism of the space-charge wave in a dielectric loaded waveguide has been demonstrated in a previous experiment. 9 Initial perturbation of the space-charge wave may be the electron beam noise near the reflex diode mesh.

Since the space-charge wave accelerates ions, simultaneously growing its amplitude by instability, the length of the whole accelerator can be considerably reduced, offering better opportunity to control the experiment. Moreover, the simultaneous growth provides a high acceleration field throughout the acceleration range. The compactness of the accelerator is similar to the ion acceleration by a linear electron beam. ${ }^{4}$ In this regard, the space-charge resonance accelerator can be very cost effective. In a practical point of view the acceleration portion in a space-charge resonance accelerator can be less than one meter. Therefore, the space-charge resonance accelerator is obviously more advantageous in comparison with the converging guide accelerator ${ }^{2}$ (CGA), where a large amplitude space-charge wave is launched from outside. The novelty of the space-charge resonance accelerator is the use of dielectric loading of waveguide to control the phase velocity of the space-charge wave, the utilization of the virtual cathode to minimize the initial phase velocity and a self growing wave by the instability mechanism. More elaborated theoretical work related to the spacecharge resonance accelerator is currently under investigation by authors and will be presented elsewhere.

\section{ACKNOWLEDGEMENTS}

It is a pleasure to acknowledge the benefit of useful discussions with Dr. M. J. Rhee. This research was supported by the Independent Research Fund at the Nava1 Surface Weapons Center.

\section{REFERENCES}

1. M.L. Sloan and W.E. Drummond, Phys. Rev. Lett. 31, 1234 (1973); E. Cornet, H.A. Davis, W.W. Rienstra, M.L. Sloan, T.P. Starke, and J.R. Uglum, Phys. Rev. Lett. 46, 181 (1981).

2. P. Sprangle, A.T. Drobot, and W.M. Manheimer, Phys. Rev. Lett. 36, 1180 (1976).

3. V. Serlin, R.J. Adler, J.A. Nation, G. Providakes, Bull. Am. Phys. Soc. 25, 1008 (1980).

4. W.W. Destler, L.E. Floyd, M.P. Reiser, Phys. Rev. Lett. 44, 70 (1980)

5. W.W. Destler, H.S. Uhm, H. Kim, and M.P. Reiser, J. App1. Phys. 50, 3015 (1979).
6. H.S. Uhm and A. Sternlieb, Phys. Fluids 23, 1400 (1980).

7. L.S. Bogdankevich and Rukhadze, Sov. Phys. Usp. 14. 163 (1971).

8. H.S. Uhm, Phys. Fluids 25, 690 (1982).

9. K.L. Felch, K.O. Busby, $\bar{R}$. W. Layman, and J.E. Wa1sh, in Physics of Quantum Electronics, V. 7 (Addison-Wes1ey, 1980) p. 301-322. 